\title{
Realization of wavelength conversion with hyperbolic secant femtosecond pulse in normal dispersion regime
}

\begin{abstract}
Realization of wavelength conversion based on second-order femtosecond dark solitons with hyperbolic secant pulse is presented. This is achieved by introducing localized dispersion perturbation along the optical fiber. We demonstrate that an initial $30 \mathrm{fs}$ second-order pulse decays to similar sub-pulses by applying perturbation using a step increment of $\beta 2$ from 6.3 to $15.75 \mathrm{ps} 2 \mathrm{~km}-1$. This shows that the realization of a 12 channel wavelength converter for femtosecond pulses is possible. Recent research shows the possibility of realizing wavelength conversion generated from picosecond solitons neglecting nonlinear effects. However, employing the same method for femtosecond pulses fails due to the manifestation of nonlinear effects. In this paper, pulse deformation under different levels of perturbation was tested, and appropriate perturbation leading to similar sub-pulses is achieved.
\end{abstract}

Keyword: wavelength conversion, anomalous and normal dispersion regime, dark soliton, higher-order soliton decay 